\title{
Efecto de la micorrización en el establecimiento de Pinus pseudostrobus en cárcavas
}

\section{Effect of mycorrhyzation in the establishment of Pinus pseudostrobus in gullies}

\author{
Mariela Gómez-Romero, ${ }^{* 1}$ Javier Villegas, ${ }^{2}$ \\ Cuauhtémoc Sáenz-Romero ${ }^{3}$ y Roberto Lindig-Cisneros ${ }^{4}$
}

\begin{abstract}
RESUMEN
Se evaluó el establecimiento de Pinus pseudostrobus Lindl., en tres condiciones de propagación y trasplante en cárcavas desprovistas de vegetación en la localidad de Atécuaro, municipio de Morelia, Michoacán. La primera condición corresponde a plantas de procedencia conocida, germinadas y propagadas en condiciones controladas (estratificadas a $4{ }^{\circ} \mathrm{C}$, germinadas a $25^{\circ} \mathrm{C}$ con $12 \mathrm{~h}$ luz y propagadas con riego cada tercer día), para contar con dos lotes de plantas, uno con inóculo y otro sin inóculo del hongo ectomicorrícico Pisolithus tinctorius (Pers.) Coker et Couch. Como una segunda condición, ambos lotes fueron trasplantados al sitio de plantación a los 15 meses de edad con adición de sustrato fértil (fibra de coco, corteza de árbol y agrolita) en las cepas. En otro sitio muy cercano, una tercera condición fue una reforestación convencional con plantas de la misma especie pero sin controlar las variables antes descritas. Se evaluó altura, diámetro a la altura de la base, cobertura, número de ramas y porcentaje de supervivencia. Los resultados muestran que los pinos que fueron plantados bajo las primeras condiciones presentaron mayor crecimiento y supervivencia que los pinos de la segunda reforestación (tercera condición) $(P<000,1)$. La supervivencia de los pinos inoculados fue de $86 \%$, la de la misma plantación sin inóculo $62 \%$ y la de la segunda reforestación $30 \%$. La inoculación se plantea como una estrategia a aplicar en proyectos de restauración de sitios severamente degradados y erosionados, planteándose la necesidad de una inversión que incremente el éxito en los programas de reforestación.
\end{abstract}

PALABRAS CLAVE

Erosión, Pisolithus tinctorius, propagación, reforestación, restauración ecológica.

\begin{abstract}
The establishment of Pinus pseudostrobus Lindl. was evaluated in three conditions of propagation and transplantation in gullies devoid of vegetation in the locality of Atécuaro, Municipality of Morelia, Michoacán. The first condition correspond to plants of known provenance, germinated and propagated under controlled conditions (stratified at $4{ }^{\circ} \mathrm{C}$, germinated at $25^{\circ} \mathrm{C}$ with $12 \mathrm{~h}$ of light and watered every other day), with the aim of having plants without mycorrhizae and with the ectomycorrhizal fungus Pisolithus tinctorius (Pers.) Coker et Couch. As a second condition, both groups where transplanted at 15 Michoacana de San Nicolás de Hidalgo (UMSNH), Morelia, Michoacán, México. Instituto de Investigaciones Químico Biológicas, UMSNH, Morelia, Michoacán, México. Instituto de Investigaciones Agropecuarias y Forestales. UMSNH, Morelia, Michoacán, México. Laboratorio de Ecología de Restauración. Centro de Investigaciones en Ecosistemas (CIEco), Morelia, Michoacán, México.

* Autor para correspondencia: C.e.: mariela_gomez@enesmorelia.unam.mx; margrbio13@hotmail.com
\end{abstract}


months of age with the addition of a fertile substrate (coconut fiber, bark and agrolite). In a nearby site, a third condition was a reforestation with plants of the same species but without controlling the aforementioned variables. The results show that the pine trees that were planted in the first condition performed better and had a higher survival than plants in the second reforestation (third condition) $(P<000,1)$. The survival of inoculated pines was $86 \%$, in the absence of the fungus was $62 \%$ and $30 \%$ in the second reforestation. Inoculation is a good strategy for restoration projects in severely degraded and eroded sites, although it increase the costs it also increases survival and growth.

\section{KEY WORDS}

Erosion, Pisolithus tinctorius, propagation, reforestation, ecological restoration.

\section{INTRODUCCIÓN}

Por el creciente deterioro de los recursos forestales en México, existe la necesidad de reforestar y restaurar eficientemente, tanto con fines comerciales como para recuperación de suelos (Cetina-Alcalá et al., 1999). El estado de Michoacán posee importantes recursos forestales por su cantidad, diversidad e importancia económica. Ocupa el tercer lugar nacional en producción de madera (aproximadamente $1 \times 10^{6} \mathrm{~m}^{3} / a n ̃ o$, después de Chihuahua y Durango) y el primer lugar nacional en producción de resina (35 000 t/año), no obstante que ocupa el sexto lugar nacional en existencias maderables (COFOM, 2001). En cuanto a riqueza de especies, Michoacán tiene el quinto lugar nacional en biodiversidad (COFOM, 2001) con 4831 especies vegetales. Destaca, entre otros, el género Pinus con 16 especies (Conabio et al., 2005). Se ha publicado que Pinus pseudostrobus Lindl., es una de las especies forestales de mayor importancia en México. Es un árbol de $15 \mathrm{~m}$ a $25 \mathrm{~m}$, se encuentra en laderas con elevaciones de $1600 \mathrm{msnm}$ a $3200 \mathrm{msnm}$ con temperatura de $9{ }^{\circ} \mathrm{C}$ a $40{ }^{\circ} \mathrm{C}$ con media de $14{ }^{\circ} \mathrm{C}$, con precipitaciones de $500 \mathrm{~mm}$ a 2000 mm con media de 1000 mm (Cruz de León, 1991). Se utiliza para la restauración de suelos degradados (Conafor, 2006), es buen productor de resina, ampliamente aprovechada en los estados del centro y sur del país. La madera es de buena calidad. Es una especie recomendable para plantaciones comerciales, también para su uso ornamental (Eguiluz, 1978). Por otro lado, en el estado de Michoacán existen serios problemas de deforestación; se calcula que se pierden entre 30000 ha y 40000 ha de bosque por año. Las principales causas de la deforestación son: cambio de uso del suelo, incendios forestales y tala ilegal (COFOM, 2001). En un estudio realizado con la finalidad de hacer una evaluación sobre los programas de reforestación en Michoacán y proponer estrategias que aumenten el éxito de establecimiento (Sáenz-Romero y Lindig-Cisneros, 2004), se menciona que existen serios problemas en cuanto al proceso de plantación y mantenimiento de las reforestaciones (Lindig-Cisneros et al., 2005), disminuyendo significativamente la supervivencia de las plantas. Entre los principales problemas detectados se encuentran la técnica de plantación (falta de capacitación del personal de campo, falta de herramientas adecuadas y falta de preparación del terreno previo a la plantación), fecha inadecuada de plantación (temporada de lluvias avanzada, limitando a las plantas para establecer un sistema radical suficiente, antes del inicio de la temporada de sequía) y a la pobre calidad de la planta, que representa hasta $15 \%$ de la mortalidad (Nienstaedt, 1994; Sáenz-Romero y Martínez-Palacios, 2000).

Por otro lado, se ha documentado que en la restauración ecológica se debe dirigir al ecosistema a través de una serie de fases hasta recuperar su estructura, la composición de especies y su función original (Martínez, 1996). En las estrategias de restauración de suelos degradados, es 
determinante el uso de la microbiota del suelo (Haselwandter, 1997; Requena et al., 2001; Álvarez, 2009), en especial de hongos formadores de micorrizas, ya que previenen la erosión del suelo al ser un factor en la formación de agregados y en la acumulación de materia orgánica. De esta manera, le dan estructura al suelo y reducen o evitan el efecto de la erosión tanto hídrica como eólica, así como la consecuente pérdida de nutrientes. Además, la asociación micorrícica otorga ventajas para el establecimiento de las plantas ante condiciones estresantes, como la habilidad de la planta hospedera para resistir a las condiciones de sequía (Haselwandter, 1997; Álvarez, 2009). La micorrización de varias especies de angiospermas y gimnospermas con hongos ectomicorrícicos ha tenido éxito, sobre todo en experimentos in vitro $y$, en menor cantidad, en condiciones de vivero. Entre los géneros más utilizados se encuentran Hebeloma, Laccaria, Rhizophogon, Scleroderma, Suillus y Pisolithus (Pera y Parladé 2005). Por otra parte, la inoculación en vivero para plantas destinadas a la repoblación, se ha realizado con especies cosmopolitas, como Sclerodema sp. y Pisolitus tinctorius (Valdés et al., 2010). El interés en utilizar Pisolithus tinctorius, se deriva de su éxito en la inoculación de plantas forestales en países de los cinco continentes. Las plantas con las que $P$. tinctorius establece simbiosis ectomicorrícica incluyen más de veinte géneros de gimnospermas y angiospermas con distribución mundial; incluyendo especies forestales de las familias Casuarinaceae, Dipterocarpaceae, Pinaceae, Myrtaceae y Oleaceae (Pérez-Moreno y Read, 2004; Ambriz et al., 2010). El cuerpo fructífero de este hongo mide entre $5 \mathrm{~cm}$ y $20 \mathrm{~cm}$ de altura y hasta $10 \mathrm{~cm}$ de diámetro, fibroso de color pardo amarillento, esporas de color marrón canela, globosas de $7 \mu$ a $12 \mu$ y espinas de hasta $2 \mu$ de largo. Fructifica al comienzo del otoño. Un aspecto muy importante es que puede sobrevivir en suelos empobrecidos o terrenos perturbados, así como en suelos de alta acidez, o con altas concentraciones de metales pesados y resiste periodos de estrés porsequía(García-Rodríguez et al., 2009).

\section{OBJETIVOS}

Evaluar las plantas de Pinus pseudostrobus en un experimento en condiciones de restauración ecológica en presencia/ ausencia de inoculación del hongo ectomicorrícico Pisolithus tinctorius. Comparar las plantas en condiciones de restauración con las de una reforestación convencional, con la finalidad de identificar las estrategias que mejoren la supervivencia y desempeño de las plantas para su establecimiento en un sitio severamente degradado y de esa forma recuperar una cobertura vegetal.

\section{MATERIALES Y MÉTODO}

\section{Área de estudio}

El área de estudio se ubica en el paraje conocido como Huertitas, ejido de Atécuaro, municipio de Morelia en Michoacán (Fig. 1a), lugar desprovisto de vegetación en donde se dan altas tasas de erosión en suelos acrisoles (acrisol ócrico), y existen numerosas cárcavas en al menos $6 \%$ de la superficie total. Presenta pendientes de $18 \%$ en promedio, por lo que es muy sensible a la erosión hídrica. El resto del paisaje está calculado en $46 \%$ de pastizales y $28 \%$ de tierras de cultivo (Duvert et al., 2010). La erosión en cárcavas, como consecuencia de la deforestación y técnicas de cultivo inadecuadas, han sido identificadas en más de $65 \%$ de la superficie de los suelos del país (Covaleda, 2008), por lo que las consecuencias de la degradación acelerada ha dado como resultado suelos sin capacidad de mantener la vida 
vegetal. Un ejemplo claro se presenta en los suelos acrisoles; según la FAO-ISRIC (2006), estos suelos han sido clasificados como suelos evolucionados, que se han desarrollado principalmente sobre rocas muy ácidas o arcillas muy meteorizadas, que sufren posterior degradación. Se caracterizan por presentar un contenido mayor de arcillas en los horizontes más profundos como resultado de procesos pedogenéticos. Los acrisoles tienen, a ciertas profundidades, una baja saturación de bases y arcillas de baja actividad. Por lo general, estos suelos son arcillosos y tienden a compactarse cuando carecen de cobertura vegetal, impidiendo la infiltración del agua (Covaleda, 2008).

La zona presenta un clima subhúmedo, con lluvias en verano, temperatura media anual de $13,8{ }^{\circ} \mathrm{C}$ y precipitación media anual de $1000 \mathrm{~mm}$ con una descarga media durante la época de secas de 0,05 Is $^{-1}-1,0 \mathrm{Is}^{-1} \mathrm{y}$ en la época de lluvias de $10 \mathrm{ls}^{-1}$ - $30 \mathrm{ls}^{-1}$, una altitud de $2150 \mathrm{msnm}$ - 2450 msnm (Duvert et al., 2010).

\section{Establecimiento de pruebas de restauración}

\section{Plantación sitio A}

En el año 2009 se estableció un experimento con fines de restauración con Pinus pseudostrobus. Las semillas procedentes de la región (Nuevo San Juan Parangaricutiro) se estratificaron a $4{ }^{\circ} \mathrm{C}$ (como tratamiento pregerminativo) en condiciones controladas, fueron desinfectadas previamente con hipoclorito de sodio comercial al $20 \%$ ( $\mathrm{NaClO} 20 \mathrm{ml}: 80 \mathrm{ml} \mathrm{H}_{2} \mathrm{O}$ ), y posteriormente se colocaron en cajas de Petri con papel filtro como sustrato humedecido con $2 \mathrm{ml}$ de agua destilada. Las cajas fueron selladas con parafilm $(R)$ y permanecieron durante 15 días a una temperatura de $4{ }^{\circ} \mathrm{C}$. Una vez transcurrido este tiempo, se trasladaron a una cámara de creci- miento a una temperatura constante de $25^{\circ} \mathrm{C}$ con 12 horas luz, manteniendo la humedad constante durante el proceso de germinación que fue de 21 días. Después de este periodo, se realizó el trasplante una vez que la radícula ya estaba emergida. Las plántulas fueron propagadas en una casa de sombra en contenedores rígidos de plástico medianos $\left(350 \mathrm{~cm}^{3}\right)$, se utilizó un sustrato comercial a base de fibra de coco, corteza de árbol y agrolita mezclado con arena en combinación 1:1 y esterilizado. A las cuatro semanas de edad, la mitad de las plantas de pinos fueron inoculadas con 500000 esporas del hongo ectomicorrícico Pisolithus tinctorius (el inóculo fue adquirido de manera comercial Biosyneterra Solutions Inc. L'Assomption, Quebec, Canadá) por cada planta. Formando dos tratamientos, uno de plantas inoculadas y otro de plantas sin inoculación, se consideraron 90 réplicas por cada tratamiento en un diseño completamente al azar. Posteriormente, a los tres meses de edad, se realizó un trasplante a bolsa de vivero de $600 \mathrm{~cm}^{3}$, con mayor cantidad de sustrato y se mantuvieron en casa de sombra durante 11 meses más, con riego constante cada tercer día. Posteriormente, las plantas se sacaron de la casa de sombra para su endurecimiento durante quince días antes de ser llevadas a campo.

Una vez que inició la época de lluvias, las plantas fueron transportadas a campo en cajas perforadas de plástico para facilitar el traslado hasta el sitio de estudio, donde permanecieron durante un día antes de ser trasplantadas. En la cepa de $40 \mathrm{~cm}^{3}$ se adicionó $1 / 2$ litro del medio de cultivo antes descrito y se realizó el trasplante a los 15 meses de edad de las plantas, con el cuidado suficiente para que las raíces no quedaran dobladas o expuestas y se cubrió bien la cepa con sustrato del sitio en un diseño completamente al azar con 90 réplicas por tratamiento. 


\section{Plantación sitio $B$}

En el mismo año se localizó otra plantación en una cárcava aledaña muy cercana (177 m) con condiciones muy similares de pendientes, en el mismo tipo de sustrato y plantadas al mismo tiempo (Fig. 1b). La plantación con Pinus pseudostrobus se trataba de una reforestación convencional, de las que se hacen anualmente en la región. Sin embargo, se desconoce la edad de las plantas al tiempo del trasplante, así como las condiciones de almacenamiento de la semilla, germinación, propagación o endurecimiento de la planta previo a la reforestación, incluso, la procedencia de las semillas (mismas condiciones de los programas de reforestación anuales). Se evaluaron también 90 plantas para que fueran comparables con las del experimento de restauración. Se sabe que, dadas las condiciones de las planta-
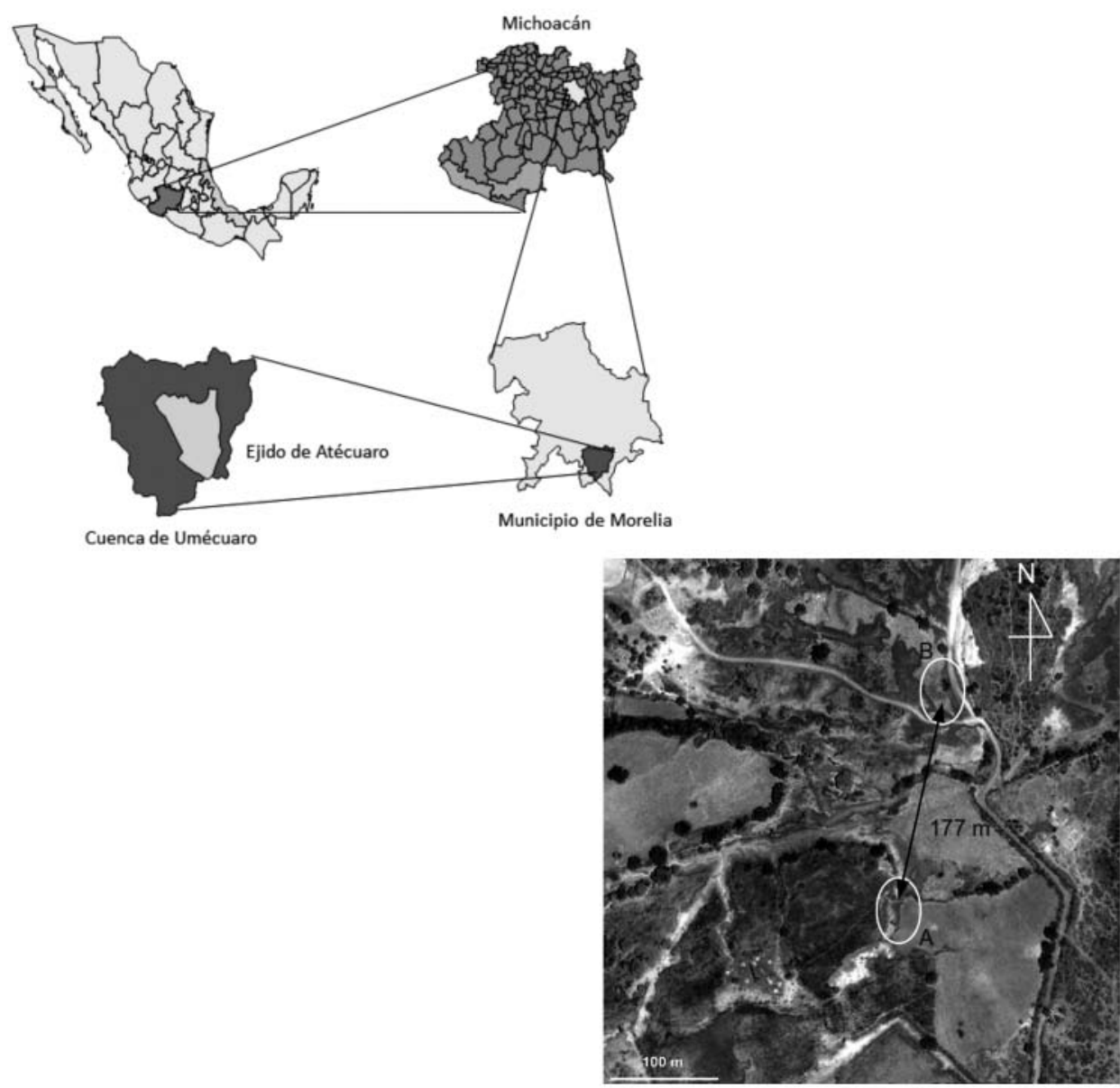

Figura 1a. Ubicación del área de estudio. 1b Sitios de estudio en la localidad de Atécuaro, municipio de Morelia, Michoacán. A) Sitio donde se encuentran los experimentos de restauración. B) Sitio donde se encuentra la reforestación convencional. 
ciones en dos sitios diferentes, ocurre que si se consideraran todos los datos como un solo experimento, un tratamiento completo (Sitio B) se encuentra en un sitio diferente, lo que corresponde a una pseudorréplica sensu Hurlbert (1984). A pesar de lo anterior se incluyeron los datos del sitio $B$ por ser una comparación útil (como testigo), pero se debe reconocer que las diferencias podrían deberse a factores inherentes a los dos sitios y no a otras diferencias.

\section{Evaluaciones}

Se realizó la evaluación de altura total, diámetro a la altura de la base (DAB) con un vernier tomada en centímetros, cobertura en $\mathrm{cm}^{2}$ obtenida a partir de la evaluación de dos diámetros de la copa e inferida mediante la fórmula de la elipse $\left(r_{1} r_{2} \pi\right)$, número de ramas y supervivencia a las plantas al tercer año de edad de establecidas las plantaciones de restauración en presencia/ausencia de inoculación con Pisolithus tinctorius (sitio A), y a las plantas de la reforestación convencional (sitio $B$ ). Los datos fueron analizados mediante Análisis de Varianza (ANOVA) en el paquete estadístico R ( $R$ Development Core Team, 2011). El análisis principal se realizó tomando en cuenta inicialmente sólo los datos del experimento del sitio $A$, al ser éste un diseño estadísticamente robusto $\mathrm{y}$, de manera adicional, un segundo análisis, consistió en comparar una plantación de reforestación convencional con la de restauración. Para analizar la supervivencia se siguió la misma estrategia, pero a través de análisis de modelos lineales generalizados (GLM) para datos con distribución binomial.

\section{RESULTADOS}

Las plantas se desempeñaron de manera diferente dependiendo de la forma en que fueron propagadas y trasplantadas en el sitio. Al comparar las plantas de la reforestación en el sitio A, en función de la presencia/ausencia de inóculación con el hongo ectomicorrízico Pisolithus tinctorius, no se obtuvieron diferencias significativas para variables de crecimiento (altura $F_{(1,267)}=$ $0,27, P=0,604$, diámetro a la altura de la base $F_{(1,267)}=2,45, P=0,119$, cobertura $F_{(1,267)}=0,782, P=0,779$ y ramas $F_{(1,267)}=$ $2,42, P=0,12)$. Sin embargo, para la supervivencia sí se presenta un incremento significativo en las plantas inoculadas. Por otro lado, al hacer la comparación de las plantaciones de los sitios A y B se presentaron diferencias estadísticamente significativas en todas las variables de crecimiento y en supervivencia.

En términos de la altura, cuando se analizan sólo los tratamientos del experimento de inoculación no hay diferencias significativas, pero cuando se incluyen los datos de las plantas de la reforestación convencional (sitio B), sí se presentan diferencias estadísticamente significativas $\left(F_{(2,267)}=87,87, P<0,0001\right)$. La altura de los tratamientos de restauración en presencia/ausencia de hongos micorrícicos $(80 \mathrm{~cm})$, contrasta con las plantas de la reforestación convencional $(40 \mathrm{~cm})$ (Fig. 2). Para el DAB se presentó un patrón similar, entre tratamientos de inoculación no se presentaron diferencias, pero sí entre las plantas del sitio $A$ y las del sitio $B$ $\left(F_{(2,267)}=233,75, P<0,00001\right)$. Los valores de las plantas de la restauración son de alrededor de $3 \mathrm{~cm}$, mientras que para las de la reforestación es menor a $1 \mathrm{~cm}$ (Fig. 3). Para la cobertura, se presentan diferencias altamente significativas entre los tratamientos del experimento de inoculación con los de la reforestación en el sitio B $\left(F_{(2,267)}=147,07, P<0,0001\right)$, con tendencias muy similares a las de la altura, con valores similares entre las plantas de los tratamientos de restauración $\left(40 \mathrm{~cm}^{2}\right)$ pero muy diferentes a las plantas de la reforestación $\left(10 \mathrm{~cm}^{2}\right)$ (Fig. 4). Para el 


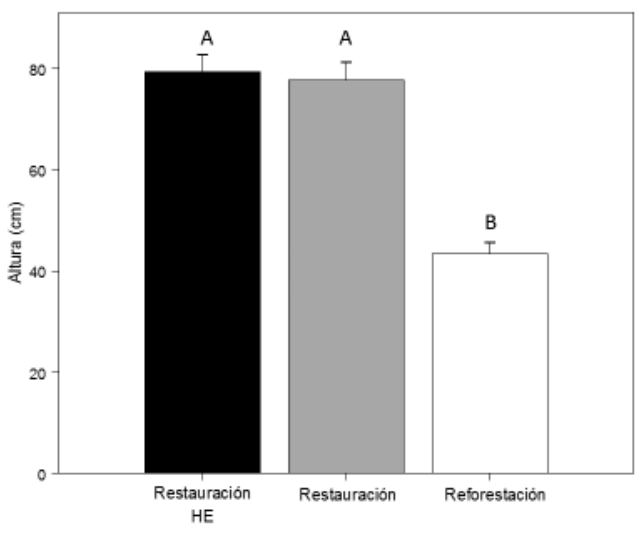

Figura 2. Altura de Pinus pseudostrobus en condiciones de restauración ecológica con inoculación de hongo ectomicorrícico (Pisolithus tinctorius) (HE), restauración sin inoculación y reforestación convencional. Las barras muestran el error estándar y las letras grupos de acuerdo con la prueba de Tukey.

Figura 3. Diámetro de Pinus pseudostrobus en condiciones de restauración ecológica con inoculación de hongo ectomicorrícico

(Pisolithus tinctorius) (HE), restauración sin inoculación y reforestación convencional. Las barras muestran el error estándar las letras grupos de acuerdo con la prueba de Tukey.
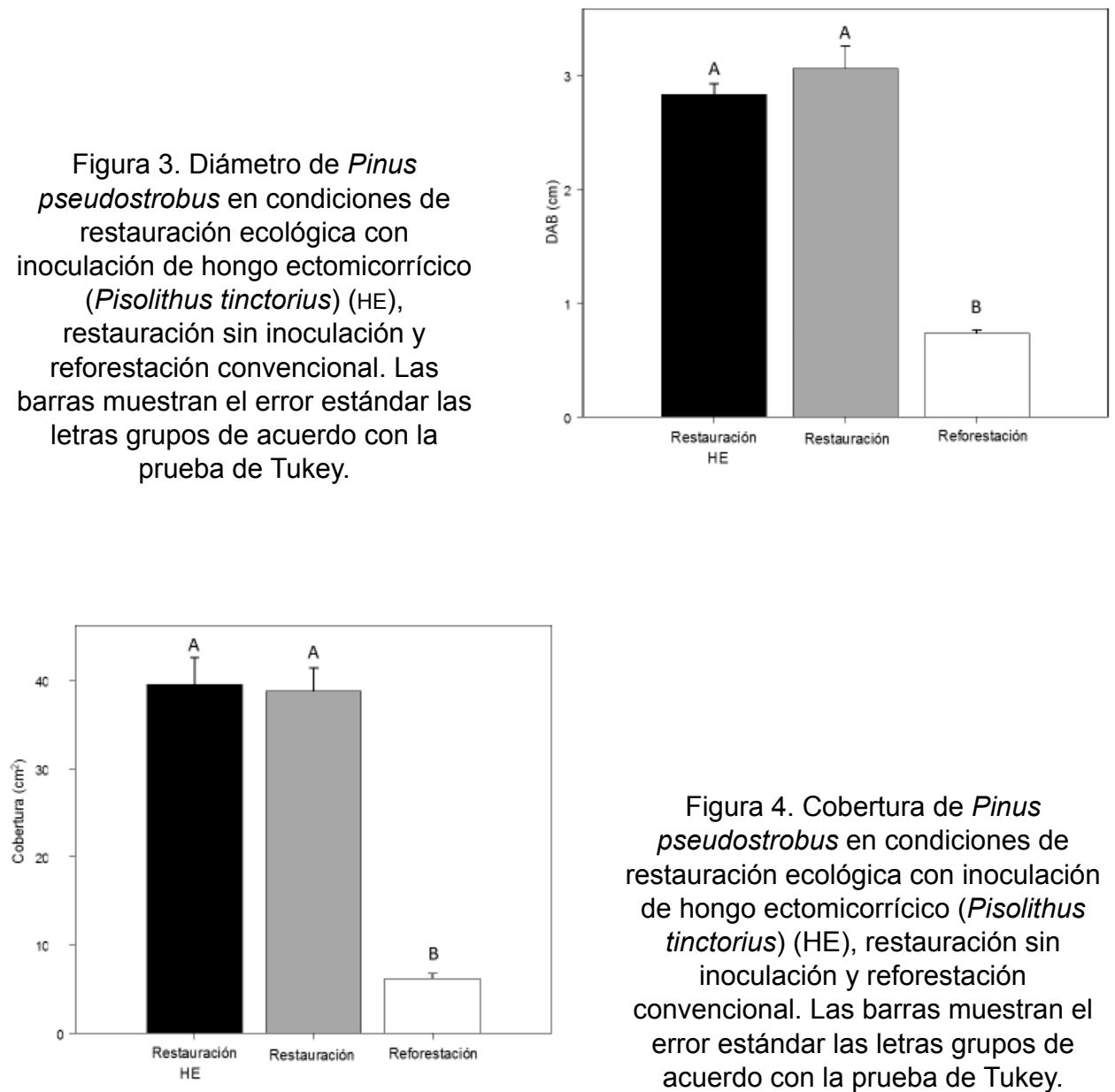

Figura 4. Cobertura de Pinus pseudostrobus en condiciones de restauración ecológica con inoculación de hongo ectomicorrícico (Pisolithus tinctorius) (HE), restauración sin inoculación y reforestación convencional. Las barras muestran el error estándar las letras grupos de acuerdo con la prueba de Tukey. 
número de ramas, se presentan tendencias muy similares, con valores mayores en las plantas del experimento en el sitio A en ausencia de hongos micorrícicos, no siendo diferente de los que se encuentran en presencia de hongos micorrícicos, pero sí estos dos de las plantas de la reforestación en el sitio $\mathrm{B}\left(F_{(2,267)}=232,78, P<\right.$ 0,0001) (Fig. 5).

El resultado más interesante se da en términos de los porcentajes de supervi-

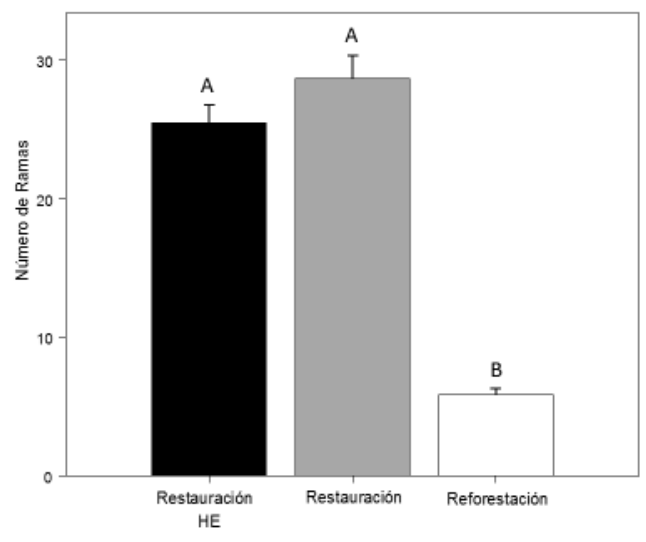

vencia; para el experimento de inoculación, las plantas inoculadas presentaron $86 \%$ de supervivencia, en ausencia de inóculo $62 \%$, siendo una diferencia en supervivencia estadísticamente significativa $\left(X^{2}=13,053\right.$, g.I. $\left.=1 ; P=0,0003\right)$. Si se incluye en el análisis a las plantas presentes en el sitio B correspondientes a las de una reforestación convencional, éstas presentaron $30 \%$ de supervivencia al tercer año del trasplante. Al comparar con los tratamientos de inoculación, existe una

Figura 5. Número de ramas de Pinus pseudostrobus en condiciones de restauración ecológica con inoculación de hongo ectomicorrícico (Pisolithus tinctorius) (HE), restauración sin inoculación y reforestación convencional. Las barras muestran el error estándar las letras grupos de acuerdo con la prueba de Tukey.
Figura 6. Supervivencia de Pinus pseudostrobus en condiciones de restauración ecológica con inoculación de hongo ectomicorrícico (Pisolithus tinctorius) (HE), restauración sin inoculación y reforestación convencional. Letras diferentes indican diferencias significativas con $p<0,05$.

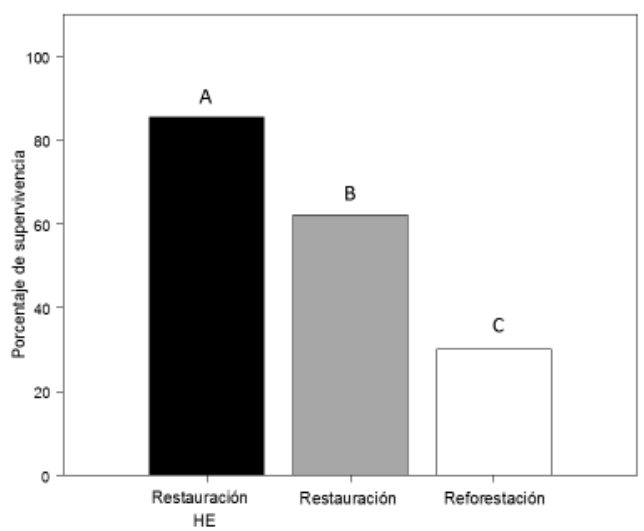


diferencia altamente significativa entre los tres $\left(X^{2}=61,366\right.$, g.I. $=2$; $P<0,0001$; Fig. 6).

\section{DISCUSIÓN}

Los resultados del experimento sugieren que, en condiciones de restauración, con estrés nutricional e hídrico, en cuanto a las variables estudiadas, las plantas inoculadas con el hongo ectomicorrícico Pisolithus tinctorius, no responden de manera diferente que las plantas sin inóculo. Sin embargo, sí les confiere beneficios para la adquisición de nutrientes que les provee de mayor supervivencia (86\%), lo cual implica una gran ventaja para el establecimiento de las plantas ante estas condiciones. Las plantas en ausencia del inóculo presentaron $62 \%$. Por otro lado, aun cuando la asociación simbiótica puede representar un gasto energético para los pinos, debido a que tienen que intercambiar hidratos de carbono (Choi et al., 2005), la relación es positiva, ya que los hongos micorrícicos proveen de nutrientes y agua a la planta, además de que le ayudan a amortiguar cambios bruscos de temperatura (Smith y Read, 2000).

La disponibilidad de agua tiene el efecto directo de disminuir la turgencia, e indirectamente limita el incremento de biomasa, por lo que una cantidad adecuada de agua disponible es esencial para mantener la productividad de las plantas (Montoya, 2000), de modo que la presencia de micorrizas puede influir de manera importante para tener disponibilidad de agua e incrementar la biomasa, sobre todo en la parte radical. El sustrato, rico en materia orgánica que se adicionó a las plantas del experimento de restauración, muy probablemente también contribuyó a generar los patrones observados, ya que a las plantas de la reforestación convencional no se les suministró ningún sustrato adicional y la materia orgánica del suelo con- tribuye a un mejor desempeño de las micorrizas. En estas condiciones de reforestación convencional las plantas presentaron $30 \%$ de supervivencia.

Arias (1992), menciona que P. pseudostrobus plantado bajo buenas condiciones de fertilidad, presenta alta tasa de crecimiento, pero también se ha publicado que no puede prosperar en sitios con condiciones edáficas adversas (DomínguezCaballero et al., 2001). El presente estudio sugiere que aplicando estrategias adecuadas, esta especie puede mostrar un buen desempeño temprano, aun sobre cárcavas. Pera y Parladé (2005) sugieren que la utilización de plantas micorrizadas pueden jugar un papel importante en la reforestación de zonas fuertemente alteradas. La inoculación con hongos micorrícicos puede incrementarsignificativamente la supervivencia de Pinus pseudostrobus como se muestra en este experimento.

Valdés et al. (2010) mencionan que la inoculación con Pisolithus tinctorius (cepa 202) a los 18 meses puede incrementar significativamente el volumen y biomasa $\left(2,93 \mathrm{~cm}^{3}\right.$ y $\left.3,68 \mathrm{~g}\right)$ de $P$. pseudostrobus con respecto al control $\left(2,3 \mathrm{~cm}^{3}\right.$ y $3,39 \mathrm{~g}$ ). Por otro lado, se han realizado experimentos con esta especie de hongo ectomicorrícico ( $P$. tinctorius) inoculando plantas de Cistus albidus en condiciones de campo y mencionan que la inoculación tiene un efecto positivo sobre el crecimiento de las plantas, obteniendo hasta $30 \%$ mayor crecimiento que en las plantas control (Alguacil et al., 2003).

En experimentos con Pinus greggii inoculados con los hongos ectomicorrícicos Laccaria laccata, Suillus pseudobrevipes, Boletus pinophilus y Pisolithus tincorius, Carrera-Nieva y López-Ríos (2004) encontraron diferencias estadísticamente significativas a los nueve meses de inoculación en la altura y biomasa en peso seco de la planta, independiente- 
mente del hongo que utilizaron, para la variable diámetro, el hongo que presentó un mayor efecto fue $B$. pinophilus. En el mismo estudio, para la especie Pinus patula utilizaron las especies Laccaria laccata, Suillus pseudobrevipes, Boletus clavipes, Amanita fulva y Amanita caesarea, encontrando que $L$. laccata es el hongo que presenta un mayor efecto en las variables altura, diámetro y biomasa en peso seco. Por otro lado, en estudios con Pinus ponderosa inoculados con los hongos micorrícicos Suillus luteus, Hebeloma mesophaeum y Rhizopogon roseolus, Barroetaveña et al. (2012) no encontraron diferencias estadísticamente significativas en el diámetro ni en el largo del vástago, comparativamente con las plantas control.

La respuesta puede deberse tanto a la especie del hongo ectomicorrícico, como a la especie de la planta hospedera debido al reconocimiento en la asociación.

Debido a que ambos sitios se encuentran a una corta distancia (177 m en línea recta), los resultados sugieren que las diferencias entre las plantas del sitio $A$, el experimento de inoculación y del sitio $B$ se deben a los tratamientos aplicados, aun cuando es posible que se deban a diferencias microambientales como la temperatura, la luz disponible o la humedad (Prince et al., 2001), que no fue posible medir en el presente estudio.

Tomar en cuenta todos los aspectos que se mencionan tanto para la germinación como para la propagación, implica una inversión adicional de recursos económicos que, sin embargo, se compensa por la mejor supervivencia y desempeño general de la plantación y que incluso puede ser calculado (García-Frapolli y Lindig-Cisneros, 2011).

Si no se cuenta con recursos adicionales, se puede reducir el área refores- tada y asignar los recursos ahorrados a estas medidas adicionales; esta estrategia incluso permitiría contar con una mayor área reforestada en el largo plazo (Sáenz-Romero y Lindig-Cisneros, 2004).

\section{CONCLUSIONES}

La inoculación con el hongo ectomicorrícico Pisolithus tinctorius incrementa la supervivencia de Pinus pseudostrobus en sitios desprovistos de vegetación, con numerosas cárcavas y graves problemas de erosión.

Por otro lado, el conjunto de condiciones a las que son sometidas las plantas durante la germinación (tratamiento pregerminativo), propagación (sustrato, endurecimiento) y trasplante (edad de las plantas, fecha y método de plantación) son de vital importancia para lograr el éxito en su establecimiento, supervivencia y crecimiento. El desempeño de las plantas (altura, diámetro, cobertura y número de ramas) independientemente de si las plantas fueron inoculadas o no, fue superior a las de la reforestación convencional.

Lo más sobresaliente del presente ensayo fue que las plantas inoculadas, presentaron un porcentaje de supervivencia mayor, seguidas de las plantas no inoculadas pero propagadas bajo las mismas condiciones que las primeras y muy por debajo los pinos de la reforestación convencional. Pinus pseudostrobus inoculado con Pisolithus tinctorius tiene potencial para reforestar sitios severamente degradados con problemas de erosión y formación de cárcavas, como es el caso en este sitio de estudio. El conjunto de estrategias representan un gran potencial de restauración ecológica y, si las tasas de crecimiento a mediano plazo fueran satisfactorias, también para el establecimiento de plantaciones con fines de producción. 


\section{REFERENCIAS}

Alguacil, M.M., F. Caravaca, R. Azcón, J. Pera, G. Díaz y A. Roldán. 2003. Improvements in soil quality and performance of mycorrhizal Cistus albus L. seedlings resulting from addition of microbially treated sugar beet residue to a degraded semiarid Meditarranean soil. Soil Use and Management 19:277-283.

Álvarez, J.F. 2009. Ecología de micorrizas arbusculares y restauración de ecosistemas. Las prensas de ciencias. Universidad Nacional Autónoma de México. México. 360 p.

Ambríz, E., A.L Báez-Pérez, J.M. Sánchez-Yáñez, P. Moutoglis y J. Villegas.2010.Fraxinus-Glomus-Pisolithus symbiosis: Plant growth and soil aggregation effects. Pedobiología 53:369-373.

Arias, H. 1992. Evaluación de la reforestación sobre tepetates en la zona oriente de la cuenca de México. Tierra. Número especial: Suelos volcánicos endurecidos. México. 10:309-317.

Barroetaveña, C., V.N. Bassani y M. Rajchenberg. 2012. Inoculación micorrícica de Pinus ponderosa en la Patagonia Argentina: colonización de las raíces, descripción de morfotipos y crecimiento de las plántulas en vivero. Bosque 33(2):163-169.

Cetina-Alcalá, V., V. González-Hernández y J. Vargas-Hernández. 1999. Manejo en vivero de Pinus greggii Engelm. y la calidad de planta. Agrociencia 33(4):423-430.

Choi, D.S., A.M. Quoreshi, Y. Maruyama, H.O. Jin y T. Koike. 2005. Effect of ectomycorrhizal infection on growth and photosynthetic characteristics of
Pinus densiflora seedlings grown under elevated $\mathrm{CO}_{2}$ concentrations. Photosynthetica 43 (2):223-229.

COFOM (Comisión Forestal del Estado de Michoacán). 2001. Atlas Forestal del Estado de Michoacán. Morelia, Michoacán, México, $97 \mathrm{p}$.

Conabio (Comisión Nacional para el Conocimiento y Uso de la Biodiversidad). Gobierno del Estado de Michoacán, SUMA (Secretaría de Urbanismo y Medio Ambiente) y UMSNH (Universidad Michoacana de San Nicolás de Hidalgo). 2005. La Biodiversidad en Michoacán. Estudio de Estado. 266 p.

Conafor (Comisión Nacional Forestal). 2006. Pinus pseudostrobus http:// www.conafor.gob.mx/programas nacionales_forestales/pronare/ Fichas\%20Tecnicas/Pinus\%20pseudostrobus.pdf.

Carrera-Nieva, A. y G.F. López-Ríos. 2004. Manejo y evaluación de ectomicorrizas en especies forestales. Revista Chapingo. Serie Ciencias Forestales y del Ambiente. 10(2):93-98.

Covaleda, O.S. 2008. Influencia de diferentes impactos antrópicos en la dinámica del carbono y la fertilidad de suelos volcánicos mexicanos, implicaciones sobre el secuestro de carbono. Tesis doctoral. Escuela Técnica Superior de Ingenierías Agrarias, Departamento de Ciencias Agroforestales. Universidad de Valladolid. Palencia, España. 367 p.

Cruz de León, J. 1991. Untersuchung des technologischen Gebrauchswertes der mexikanischen Kiefernart Pinus pseudostrobus Lindley. Göttinger Beiträge Zur Land - und Forstwirtschaft in den Tropen und Subtropen 66:1-185. 
Domínguez-Caballero, P.A., J.J. NavarChaidez y J.A. Loera-Ortiz. 2001. Comparación del rendimiento de pinos en la reforestación de sitios marginales en Nuevo León. Madera y Bosques 7(1):27-35.

Duvert, C., N. Gratiot, O. Evrard, O. Navratil, J. Némery, C. Prat y M. Esteves. 2010. Drivers of erosion and suspended sediment transport in three headwater catchments of the Mexican Central Highlands. Geomorphology 123:243-256.

Eguiluz, T. 1978. Ensayo de integración de conocimientos sobre el género Pinus en México. Tesis de Licenciatura para obtener el título de Ingeniero Agrónomo. Escuela Nacional de Agricultura. Chapingo, México.

FAO-ISRIC. 2006. World reference base for soil resources. A framework for international classification correlation and communication. $125 \mathrm{p}$.

García-Frapolli, E. y R. Lindig-Cisneros. 2011. Barreras e incentivos económicos para la restauración de la biodiversidad. Acta Biológica Colombiana 16:269-280.

García-Rodríguez, J.L., J. Pérez-Moreno, A. Aldrete, V.M. Cetina-Alcalá, y H. Vaquera-Huerta. 2009. Caracterización del hongo silvestre ectomicorrízico Pisolithus tinctorius (Pers.) Coker et Couch en cultivo y en simbiosis con eucalipto y pino. Agrociencia 40:665-676.

Haselwandter, K. 1997. Soil micro-organisms, mycorrhiza and restoration ecology. In: K.M. Urbanska, N.R. Webb y P.J. Edwards, eds. Restoration ecology and sustainable development. Cambridge University Press. Reino Unido. p:65-80.
Hurlbert, S.H. 1984. Pseudoreplication and the design of ecological field experiments. Ecological Monographs 54(2):187-211.

Lindig-Cisneros, R., A. Blanco-García y C. Sáenz-Romero. 2005. Estrategias para la restauración ecológica de bosques. Biológicas 7:13-21.

Martínez, R.E. 1996. La restauración ecológica. Ciencias. 43:56-61.

Montoya, F. 2000. Mejoramiento de la productividad de Eucalyptus nitens (Deane \& Maiden) Maiden, al primer año de establecimiento a través del riego y fertilización en un suelo rojo arcilloso del valle central de la VIII región. Tesis de licenciatura para la obtención del título de Ingeniero Forestal. Universidad Católica de Temuco. Escuela de Ciencias Forestales. Temuco, Chile. 60 p.

Nienstaedt, H. 1994. Reforestación en México con semilla mejorada. Boletín Mendel Sociedad Mexicana de Genética. 3(1):3-6.

Pera, J. y J. Parladé. 2005. Inoculación controlada con hongos ectomicorrícicos en la producción de planta destinada a repoblaciones forestales: estado actual en España. Investigación Agraria, Sistemas y Recursos Forestales 14(3):419-433.

Pérez-Moreno, J. y D. Read. 2004. Los hongos ectomicorrízicos, lazos vivientes que conectan y nutren a los árboles en la naturaleza. Interciencia 29:239-247.

Prince, T.D., N.E. Zimmermann, P. Van Der, M.J. Lexermeer, P. Leadley, I.T. Jorristsma, J. Schaber, D.F. Clark, P. Lasch, S. Mcnulty, J. Wu y B. Smith. 2001. Regeneration in gap models: 
Priority issues for studying forest responses to climate change. Climatic Change 3-4(51):475-508.

R Development Core Team. 2011. R: A Language and Environment for Statistical Computing. R Foundation for Statistical Computing, Vienna, Austria. ISBN 3-900051-07-0. URL: http://www.R-project.org.

Requena, N., E. Pérez-Solís, C. AzcónAguilar, P. Jeffries y J.M. Barea. 2001. Management of indigenous plant-microbe symbioses aids restoration of desertified ecosystems. Applied and Environmental Microbiology 67:495-498.
Sáenz-Romero, C. y A. Martínez-Palacios. 2000. Variación genética altitudinal de pinos. Nuestro Bosque 1(2):10-11.

Sáenz-Romero, C. y R. Lindig-Cisneros. 2004. Evaluación y propuestas para el programa de reforestación en Michoacán, México. Ciencia Nicolaita 37:107-122.

Smith, A.E. y D.J. Read. 2000. Mycorrhizal Symbiosis. 2a. ed. Academic Press. Londres. $605 \mathrm{p}$.

Valdés, M., E. Ambriz, A. Camacho y A. M. Fierros. 2010. Inoculación de plántulas de pinos con diferentes hongos e identificación visual de la ectomicorriza. Revista Mexicana de Ciencias Forestales 2(1):53-64. 